\title{
Motif Tool Manager: a web-based platform for motif discovery Vinhthuy Phan
}

\author{
Address: Department of Computer Science, The University of Memphis, Memphis, TN 38152, USA
}

Email: Vinhthuy Phan - vphan@memphis.edu

from UT-ORNL-KBRIN Bioinformatics Summit 2009

Pikeville, TN, USA. 20-22 March 2009

Published: 25 June 2009

BMC Bioinformatics 2009, I0(Suppl 7):AI7 doi:10.1 186/147|-2105-10-S7-AI7

This abstract is available from: http://www.biomedcentral.com/I47I-2/05/I0/S7/AI7

(c) 2009 Phan; licensee BioMed Central Ltd.

\section{Background}

DNA motif discovery remains an important but challenging problem for biologists and computer scientists. A contributing factor for this inherent difficulty lies in the fact that motifs are fairly short (between 5- to 20- basepairs long) and can easily be confused with random signals in the much larger promoters of order of thousands of basepairs. Consequently, while many techniques have been employed in an attempt to address this problem including combinatorial enumeration, probabilistic modeling and heuristic search, there is none that outperforms the others across different types of data and organisms and further suggested that complimentary techniques be used. Unfortunately, it is not convenient for many researchers, especially life scientists, to compare many of these methods before settling on one that satisfies their needs. We introduced Motif Tool Manager [1], which is a web-based platform for comparing and combining different approaches to discover novel DNA motifs. It comes with a set of several well-known tools for to motif discovery.

We recently added to Motif Tool Manager a new method called MotifSample [2]. Motif Tool Manager was built on top of a more general framework [3] and as such it provides an easy mechanism for adding new motif finding tools to the platform and a minimal setup of the tools on the server. Users can interact with the tools through the web-based platform and compare results from different approaches. Our system provides a basic mechanism for identifying the consensus motif candidates. URL: http:// cetus.cs.memphis.edu/motif/

\section{References}

I. Phan V, Furlotte NA: Motif Tool Manager: a web-based framework for motif discovery. Bioinformatics 2008, 24(24):2930-293I.

2. Thijs G, Lescot M, Marchal K, Rombauts S, De Moor B, Rouzé P, Moreau $Y$ : A higher order background model improves the detection of regulatory elements by Gibbs Sampling. Bioinformatics 200I, I7(12): III3-1 I22.

3. Phan V, Malasri K: BioTDMS: a general-purpose system for integrative management of bioinformatics tools and data. Int Journal of Computational Biology and Drug Design 2008, 2(I): 174-I84. 